\title{
Linx
}

Revue des linguistes de l'université Paris X Nanterre

$82 \mid 2021$

Entre vieillissement et innovation : le changement linguistique

\section{Après que suivi de l'indicatif ou du subjonctif : quelles voies de changement dans les ouvrages de référence?}

Après que followed by indicative or subjunctive: Which changes in reference

books?

\section{Sophie Piron et Nadine Vincent}

\section{OpenEdition}

Journals

Édition électronique

URL : https://journals.openedition.org/linx/8088

DOI : $10.4000 / \operatorname{linx} .8088$

ISSN : 2118-9692

Éditeur

Presses universitaires de Paris Nanterre

Référence électronique

Sophie Piron et Nadine Vincent, «Après que suivi de l'indicatif ou du subjonctif : quelles voies de changement dans les ouvrages de référence? », Linx [En ligne], 82 | 2021, mis en ligne le 15 juillet 2021, consulté le 20 juillet 2021. URL : http://journals.openedition.org/linx/8088 ; DOI : https://doi.org/ $10.4000 / \operatorname{lin} x .8088$

Ce document a été généré automatiquement le 20 juillet 2021.

Département de Sciences du langage, Université Paris Ouest 


\title{
Après que suivi de l'indicatif ou du subjonctif : quelles voies de changement dans les ouvrages de référence?
}

\author{
Après que followed by indicative or subjunctive: Which changes in reference \\ books?
}

Sophie Piron et Nadine Vincent

\section{Introduction}

Paris, 1962. Début d'année universitaire, premier cours du certificat de grammaire et philologie françaises à la Sorbonne: dans l'amphithéâtre, quatre professeurs parmi lesquels Robert Léon Wagner et René Étiemble demandent aux 400 étudiants présents d'écrire sur une feuille une phrase contenant après que. Le dépouillement se fait en séance pendant que les étudiants s'étonnent de la banalité de l'enquête. Vient alors l'annonce des résultats : à la grande stupéfaction de la majorité des candidats, c'est l'indicatif qu'il aurait fallu employer! Les professeurs, eux, exultent et prédisent que l'évolution est engagée vers le subjonctif, qui pourrait bien devenir la norme d'ici un demi-siècle ${ }^{1}$.

C'est à cette évolution que s'intéresse le présent article. Si le subordonnant après que est censé être suivi du mode indicatif, il est de plus en plus employé avec le subjonctif (Guillaume 1929, Stéfanini 1953, Wilmet 1969, Dolbec et Le Flem 1981, Canut et Ledegen 1998, etc.).

Les symptômes diminuent rapidement

$[$ Indicatif après que le patient a pris le médicament].

$[$ subjonctif après que le patient ait pris le médicament].

Nous évaluerons le vieillissement d'un emploi, celui de l'indicatif, à travers la façon dont la règle est consignée dans les ouvrages de référence. L'étude est organisée selon 
deux perspectives, l'une grammaticale et l'autre, lexicographique. L'article expose d'abord l'avis des grammaires à propos du mode régi par après que. Il établit ensuite comment la question est traitée dans les dictionnaires, qui servent souvent de première ressource aux usagers. Les deux perspectives reposent sur l'analyse d'un corpus d'ouvrages publiés en France, en Belgique et au Québec, et remontent jusqu'aux XVI ${ }^{e}$ et XVII ${ }^{\mathrm{e}}$ siècles.

L'objectif est de voir si l'évolution d'emploi est consignée et comment elle est traitée, le cas échéant. En termes plus pratiques, il s'agissait également de proposer, sur la base de cette étude, une description éclairée de après que dans le dictionnaire Usito ${ }^{2}$.

\section{Que disent les grammaires et les ouvrages généraux de linguistique?}

\subsection{Méthodologie et objectif}

Nous avons constitué un corpus de grammaires et d'ouvrages généraux de linguistique qui couvre toute la période grammaticographique, soit depuis l'émergence des grammaires au XVI $\mathrm{XI}^{\mathrm{e}}$ sièle jusqu'à aujourd'hui. Le corpus ainsi bâti compte 117 ouvrages $^{3}$. Nous n'avions pas la volonté de construire le corpus le plus large possible, mais plutôt d'obtenir un échantillon représentatif de grammaires françaises et d'ouvrages linguistiques généraux. L'ensemble se compose, par conséquent, d'une première portion, historique, qui contient la plupart des ouvrages relevés par le Corpus de textes linguistiques fondamentaux (CTLF) entre le $\mathrm{XVI}^{\mathrm{e}}$ et le $\mathrm{XX}^{\mathrm{e}}$ siècle, sans non plus s'y restreindre. Ce sous-corpus comporte 80 titres qui sont donc considérés comme fondamentaux en grammaire française. Il s'arrête en 1980, avec la dernière édition du Bon usage, du ressort de Grevisse seul. La date correspond également à la progression du courant de la grammaire moderne dans le champ des publications. La portion contemporaine du corpus compile des titres absents du CTLF parce qu'ils sont plus récents et relève, outre les ouvrages actuellement considérés comme des références dans le domaine, ceux qui sont facilement disponibles en librairie. Nous pouvons affirmer que nous dressons ainsi un portrait assez fidèle du marché grammatical contemporain. Ce sous-corpus contient 37 titres publiés entre 1984 ( $1^{\text {re }}$ édition ou impression) et aujourd'hui.

L'objectif était de couvrir le français classique et préclassique jusqu'au français contemporain à travers les témoignages qu'en proposent les ouvrages de référence. Il ne s'agissait donc pas de voir une éventuelle évolution de l'usage à travers des textes et des emplois en contexte, mais plutôt à travers les prescriptions et descriptions des grammairiens et linguistes. Nous cherchions à savoir à partir de quand l'emploi du subjonctif à la suite de après que avait été exposé dans les grammaires et quelle attitude les auteurs adoptaient face aux usages consignés. Nous avons choisi de traiter le corpus en consultant, dans les grammaires, les chapitres où l'information que nous cherchions aurait pu être présente : ceux consacrés au verbe et à sa syntaxe, et ceux dédiés aux conjonctions et à leur syntaxe. 


\subsection{Les grammaires anciennes}

Le premier constat issu des grammaires anciennes est tout simplement l'absence d'information à ce sujet. Ainsi, sur les 80 ouvrages consultés, 47 (soit un peu plus de la moitié du corpus) n'ont rien apporté sur la question qui nous occupe ${ }^{4}$. Dans les grammaires les plus anciennes, une moindre couverture des faits linguistiques n'est pas anormale. Par contre, l'absence d'information dans les ouvrages à partir du XVII ${ }^{\mathrm{e}}$ et surtout du XVIII siècle autorise à conclure que le choix du mode à la suite de après que n'est pas un élément normatif soumis à des tensions d'évolution. Sur les 80 ouvrages du corpus ancien, les $33^{5}$ qui abordent la question du mode avec après que le font de manières diverses. Une dizaine d'entre eux se contentent d'exemplifier l'usage du mode avec après que (Régnier-Desmarais 1705, Restaut 1730, Vallart 1744, Beauzée 1767, Bescherelle 1834, Landais 1835, Brelet 1899, Bruno et Bony 1908, Grevisse 1936, Wagner et Pinchon 1961), sans énoncer la règle d'emploi. Ils convergent tous vers l'indicatif, excepté Restaut (éditions de 1730 et de 1732).

Aprés que les points principaux eurent esté ainsi reglez de part \& d'autre, on n'eut pas de peine à convenir du reste. (Régnier-Desmarais $1705: 716$ )

Après que Salomon eût bâti un temple à Dieu, il se bâtit un palais pour lui. (Restaut $1730: 197 ; 1732: 366)$

L'orthographe du subjonctif (ê̂t, fût) et de l'indicatif (eut, fut) étant bien distinguée dans les tableaux de conjugaison chez Restaut ${ }^{6}$, il faut conclure que après que est suivi du subjonctif dans l'exemple proposé (eût bâti). L'exemple de Restaut $(1730,1732)$ est issu de Richelet $(1680)^{7}$, à une différence près: Richelet le consigne sans accent circonflexe (après que Salomon eut bâti un temple à Dieu). Donc, au mode indicatif. Nous émettons l'hypothèse d'une coquille dans Restaut $(1730,1732)$, passée inaperçue aux côtés du circonflexe réclamé par le verbe bâtir.

Hormis de rares signalements (Chiflet 1659, Vairasse d'Allais 1681 et Boinvilliers 1818), le mode requis par après que - soit l'indicatif - ne fait son apparition dans les grammaires qu'au début du $\mathrm{XX}^{\mathrm{e}}$ siècle. Encore est-ce initialement de manière très générale (Sudre 1907, Calvet et Chompret 1917, Radouant 1922, Lanusse et Yvon 1920, Gaiffe et al. 1936) puisqu'il n'est fait allusion qu'à certaines subordonnées temporelles ${ }^{8}$, et non précisément au subordonnant après que.

Les Conjonctions composées de Que, qui regissent l'indicatif, sont celles cy. [...] Celles qui signifient le temps: Cõme [...] Après que vous serez arrivé, que vous fustes arrivé, que vous estes venu icy. (Chiflet 1659 : 123)

On se sert ordinairement du defini composé [nous eumes achevé, j'eus appris, il eut assemblé] aprês ces Adverbes de temps : Quand, lors que, aussitôt que, d'abord que, dés que, aprês que, dès le moment, l'heure, le jour que. \&c. (Vairasse d'Allais 1681 : 204)

Les propositions de temps de simultanéité et de postériorité, indiquant une action dont l'accomplissement est réel, se construisent, par suite, avec l'indicatif [...]. (Sudre $1907: 161$ )

Quand le fait exprimé dans la subordonnée est antérieur ou simultané par rapport à celui de la principale, il s'agit d'un fait réel ou considéré comme tel, et il s'exprime à l'aide du mode indicatif. (Radouant 1922 : 260)

Le mode apparaît ensuite explicitement précisé dans les publications du corpus, essentiellement à partir des années 1930 (Maquet et al. 1908, Académie 1932, Michaut et Schricke 1934, Bruneau et Heulluy 1937, Bloch et Georgin 1937, Gougenheim 1938, Le Bidois et Le Bidois 1938, Dauzat 1947, Von Wartburg et Zumthor 1947, Cayrou 1948, 
Souché 1948). Ces ouvrages ne signalent toutefois aucune entorse à la règle dans l'usage qui leur est contemporain.

L'indicatif s'emploie après quand, lorsque, tandis que, comme, après que, dès que, depuis que, aussitôt que, parce que, vu que, attendu que, si. (Maquet et al. 1908 : 240)

L'action antérieure est marquée par les conjonctions et locutions conjonctives : après que, depuis que, dès que, aussitôt que, quand, lorsque, qui se construisent avec l'indicatif. (Académie 1932 : 228)

L'opposition avant que - après que est une opposition de sens : avant que marque que l'action de la principale est antérieure à celle de la subordonnée, après que marque que l'action de la principale est postérieure à celle de la subordonnée. L'opposition des deux conjonctions s'accompagne d'une opposition modale subjonctif - indicatif. (Gougenheim 1966 [1938] : 343)

À la fin des années vingt, ni Bauche (1928) ni Frei (1929) ne font allusion à un usage populaire du subjonctif à la suite de après que. Dans le corpus, le premier à dresser le constat d'un changement est Bally (1932) ${ }^{9}$, dans un ouvrage de linguistique. L'origine serait une confusion orthographique entre des homophones (eut, eût ; fut, fût), mais l'erreur engage un autre plan, celui de la syntaxe. Les mots de Bally condamnent clairement la construction.

Voici qu'à son tour l'imparfait du subjonctif s'acoquine avec le passé simple de l'indicatif à la troisième sing. de toutes les conjugaisons; d'où de fâcheuses ambiguïtés [...] C'est la raison pour laquelle la $3^{\mathrm{e}}$ sing. du passé défini est parfois imprimée avec circonflexe, même là où il n'y a pas de doute sur son identité : «Lorsqu'il fût parti, etc. ». Remarquons seulement que dans le cas parallèle « après qu'il fût parti... », il y a plus qu'une faute d'orthographe ; la syntaxe de " avant qu'(il fût parti) » a déteint sur celle de après que. (Bally $1932: 255$, par. 312) ${ }^{10}$

À la même époque, Le Bidois et Le Bidois (1938) notent simplement que l'emploi du subjonctif est exceptionnel, et citent Verlaine.

L'emploi du subjonctif après cette locution est tout à fait exceptionnel : «Après que j'eusse eu fini, nous fûmes invités... à une soirée » VERL. CEuvres compl. V, 241 (Le Bidois et Le Bidois 1938, vol. $2: 428$, par. 1423)

Vient ensuite une phase de jugements très sévères à l'égard du subjonctif : De Boer (1947) le signale comme un abus, la grammaire Larousse de 1962, comme une faute grossière et un laxisme.

Et cet abus [de subjonctifs dans diverses situations] n'étonne pas trop chez un auteur [Montherlant] qui se sert quelquefois du subjonctif même après quand et après après que : « Mais quelque temps après que la voiture fût en marche..., elle éclata d'un rire strident. » (p. 236) ; « Mais c'est épouvantable, gémit Mme Emilie, quand son frère l'eût mise au courant» (p. 124) [...] un subjonctif après après que est tout à fait exceptionnel [...]. (De Boer 1958 [1947] : 151, par. 274 ; 205, par. 402)

On enseigne que avant que est suivi du subjonctif et après que de l'indicatif, le subjonctif passant pour une faute grossière. Néanmoins, le subjonctif est ici un des laxismes les plus répandus : « Il est pourtant vrai qu'en 1953, quatre ans après que les communistes aient pris le pouvoir, l'évêque attendait toujours. (Étiemble) » (Chevalier et al. $1962:$ :131)

Le jugement devient ensuite moins cinglant chez Dubois et Lagane (1973), qui l'associent simplement à la langue familière.

Le subjonctif [...] dans la langue familière, avec après que (mais, dans la langue soutenue, on n'emploie que l'indicatif après cette conjonction). (Dubois et Lagane $1973: 190)$ 


\subsection{Le bon usage, ou la courroie de transmission entre les deux corpus}

Les remarques de Grevisse au fil des éditions du Bon usage constituent un très bon indicateur d'évolution. L'ouvrage se situe à cheval sur les deux portions du corpus puisqu'il fait partie des grammaires anciennes jusqu'à son édition de 1980. À partir de celle de 1986 (révisée par Goosse), il relève du volet moderne du corpus ${ }^{11}$. Les éditions de 1936, 1939 et 1946 consignent seulement l'emploi de l'indicatif.

Les propositions temporelles qui indiquent la simultanéité ou la postériorité du fait exprimé par la principale marquent un fait présent ou passé par rapport au verbe principal, c'est-à-dire un fait réel ou regardé comme tel ; par suite elles se mettent à l'indicatif : [...] Après qu'il eut brouté (...), Jeannot Lapin retourne aux souterrains séjours. (La Font.). (Grevisse 1936, par. 1003 ; 1939 et 1946, par. 1018).

Grevisse ne signale après que suivi du subjonctif qu'à partir de la $4^{\mathrm{e}}$ édition du Bon usage, soit celle de 1949. La question du mode passe désormais des ouvrages de linguistique (Bally 1932, Le Bidois et Le Bidois 1938, De Boer 1947) aux grammaires. Le bon usage modifiera deux éléments au fil du temps à l'égard du subjonctif : la fréquence d'emploi (parfois [1949]; une tendance [1953, 1955, 1961, 1964] ; une forte tendance [1969, 1975, 1980] ; tendance surtout forte depuis le deuxième tiers du XXe siècle [1986, 1993, 2007, 2011, 2016]) et le jugement normatif (construction fort suspecte d'incorrection [1949]; construction suspecte $[1953,1955,1961,1964,1969,1975]$; se résigner à admettre [1980]; tendance irrésistible [1986, 1993, 2007, 2011, 2016]). Le basculement s'opère en 1980, mais le subjonctif n'est encore admis à ce moment que du bout des lèvres.

On trouve parfois après que construit avec le subjonctif [...] il convient, en attendant que l'usage se soit nettement déclaré, de tenir cette construction pour fort suspecte d'incorrection. (Grevisse 1949 : par. 1018 a 2)

On constate, dans l'usage des journalistes et aussi dans la littérature, une tendance à construire après que avec le subjonctif. [...] il conviendrait, en attendant que l'usage se soit nettement déclaré, de tenir pour suspecte la construction de après que avec le subjonctif. (Grevisse 1953, 1955, 1961, 1964 : par. 1018 a 2)

On constate, depuis une quarantaine d'années, dans l'usage des journalistes, de la radio et de la télévision, et aussi dans la littérature, une forte tendance à construire après que avec le subjonctif. [...]. Le cas reste controversé, mais ici, l'usage, ce tyran, impose sa loi ; il faut bien se résigner, en dépit qu'on en ait, à admettre après que avec le subjonctif, tant les exemples abondent. (Grevisse 1980, par. 2614)

Malgré la règle donnée ci-dessus, on observe une tendance, surtout forte depuis le deuxième tiers $\mathrm{du} \mathrm{XX}^{\mathrm{e}}$ siècle, à faire suivre après que du subjonctif. [...] Cette tendance a fait l'objet de vives critiques [...]. Elle parait pourtant irrésistible (Grevisse 1986, par. 1082), quoique l'indicatif ne soit nullement périmé. (Grevisse 1993, par. 1083 ; 2007, 2011, 2016 par. 1137)

\subsection{Les grammaires modernes}

Le corpus est ici composé de 37 titres, s'échelonnant entre 1984 et 2020. Une proportion de 17 ouvrages (soit à peine moins de la moitié du corpus) ne souffle mot de < après que + subjonctif $>$. Tantôt les subordonnants requérant l'indicatif ne sont tout simplement pas envisagés ${ }^{12}$. Tantôt il est seulement précisé que après que se construit avec l'indicatif, mais l'insistance semble faire écho à un constat d'emploi du subjonctif ${ }^{13}$. Il s'agit de grammaires scolaires (destinées à la formation des enseignants ou au monde scolaire en général), mais aussi de grammaires normatives pour un public intéressé. 
Le mode des subordonnées de temps est l'indicatif, y compris celles introduites par après que, sauf pour celles qui expriment la postériorité et qui sont introduites par avant que, jusqu'à ce que, en attendant que, qui sont au subjonctif. (Pellat et Fonvielle $2016: 306)$

Le reste du corpus contemporain (soit un peu plus de la moitié, avec 20 titres sur 37) aborde l'emploi du subjonctif, toujours aux côtés de l'indicatif. Trois attitudes se détachent alors : la condamnation plus ou moins forte, avec ou sans détours (c'est une faute, malgré sa fréquence, c'est du français parlé : Arrivé et al. 1986, Delatour et al. 2004, Éluerd 2009, Struve-Debeaux 2010, Bescherelle 2012, Vassevière 2013, Fairon et Simon 2018), le constat que l'usage actuel n'est, de plus en plus souvent, pas conforme à la règle (Le Goffic 1993, Grevisse et Goosse 1995, Chartrand et al. 1999, Riegel et al. 2018, Narjoux 2018) et l'acceptation plus ou moins nette de cette nouvelle possibilité (Gobbe et Tordoir 1984, Maingueneau 1991, Denis et Sancier-Château 1997, Bosquart 1998, Cherdon 2005, Wilmet 2010, Grevisse et Goosse 2016, Piron 2017, Le Goffic 2019).

Peut-être par analogie avec avant que, l'emploi du subjonctif avec après que est fréquent. Il n'en est pas moins fautif. On doit dire après qu'il $a$, et jamais après qu'il ait. (Bescherelle 2012 : 275, par. 384)

La norme prescrit l'indicatif [...] mais le subjonctif est courant [...]. (Le Goffic 1993 : 437)

Or, depuis le milieu du $\mathrm{XX}^{\mathrm{e}}$ siècle, le subjonctif se rencontre de plus en plus souvent avec après que [...]. (Riegel et al. $2018: 568$ )

Après que se construit de plus en plus fréquemment avec le subjonctif dans la langue courante, et même dans la langue littéraire. (Gobbe et Tordoir 1984 : 106)

L'analogie avec les temporelles introduites par avant que conduit parfois à l'emploi $\mathrm{du}$ subjonctif dans les subordonnées introduites par après que. Cette évolution se constate de plus en plus largement dans le français courant. (Denis et SancierChâteau 1997 : 514)

La conjonction après que, traditionnellement suivie de l'indicatif, se construit de plus en plus souvent avec le subjonctif. (Bosquart 1998 : 451)

Les deux attitudes les plus répandues au sein du corpus sont la condamnation et l'acceptation, mais c'est bien cette dernière qui l'emporte. Elle représente à elle seule presque la moitié des avis sur le subjonctif, mais surtout, associée aux constats sans condamnation, elle fait basculer le subjonctif vers une forte majorité d'avis. Les ouvrages les plus réfractaires à l'emploi du subjonctif sont, soit les plus anciens, soit des ouvrages destinés à un public assez large ou scolaire (par exemple, Bescherelle 2012, Fairon et Simon 2018). À cet égard, deux ouvrages scolaires québécois (Bosquart 1998, Chartrand et al. 1999) se démarquent puisqu'ils ont opté pour l'ouverture au subjonctif.

On soulignera une certaine frilosité des grammaires à proposer des exemples au subjonctif. On en trouve ainsi seulement chez Gobbe et Tordoir (1984), Grevisse et Goosse (1995), Bosquart (1998), Riegel et al. (2018), Piron (2017) et Narjoux (2018). Le fait est plus rare dans les ouvrages où l'emploi est critiqué (Arrivé et al. 1986, Éluerd 2009, Fairon et Simon 2018).

\section{Traitement de après que dans les dictionnaires contemporains}

Nous avons étudié différents dictionnaires, et l'attestation d'une possibilité de l'emploi du subjonctif à la suite d'après que apparaît clairement dans les ouvrages de référence 
dès la seconde moitié du $\mathrm{XX}^{\mathrm{e}}$ siècle ${ }^{14}$. Nous nous concentrons donc ici sur des dictionnaires du XXe et du $\mathrm{XXI}^{\mathrm{e}}$ siècle, autant des dictionnaires en un volume qu'en plusieurs, et aussi bien des dictionnaires de langue que des dictionnaires de difficultés. Cette recension nous amène à constater l'existence de trois types de description pour après que :

- L'attestation de l'indicatif seul ;

- l'attestation de l'indicatif ou du subjonctif, avec critique du subjonctif ;

- l'attestation de l'indicatif et du subjonctif, avec acceptation du subjonctif.

Voyons plus en détail chacune de ces trois catégories.

\subsection{Le subjonctif n'est pas mentionné}

À l'image des ouvrages antérieurs au XXe siècle, Le Petit Larousse illustré 2021 est le seul dictionnaire de notre corpus qui n'indique que l'indicatif comme mode verbal pouvant être utilisé avec après que, ignorant les autres possibilités pourtant présentes dans l'usage.

APRÈS QUE loc. conj. (suivi de l'indic.) Une fois que : Après que tu auras fini. (PLI2021)

Le Petit Larousse, contenant dans un même volume les emplois communs et les noms propres, dispose d'un espace limité. Par exemple, il n'a jamais recours à des citations pour illustrer les acceptions décrites et même les exemples construits sont utilisés avec parcimonie. De plus, le Larousse indique très peu d'emplois critiqués. On peut donc supposer que même en sachant que des occurrences nombreuses existent au XXI ${ }^{\mathrm{e}}$ siècle d'après que suivi du subjonctif, les lexicographes de la maison Larousse ont choisi d'attester la seule construction qu'ils jugent de registre standard.

\subsection{Le subjonctif est critiqué}

À partir de la seconde moitié du $\mathrm{XX}^{\mathrm{e}}$ siècle, nombreux sont les ouvrages qui reconnaissent l'existence de la construction après que + subjonctif, mais qui la disent fautive. Certains vont jusqu'à attester la construction critiquée, l'illustrant même par des citations littéraires, alors que d'autres se contentent d'indiquer la prescription, et parfois d'expliquer l'erreur commise en comparant les locutions avant que et après que.

\subsubsection{Dictionnaire de l'Académie française (DAF)}

La construction avec le mode subjonctif est absente de la $8^{e}$ édition du Dictionnaire de l'Académie française (1932-1935), mais il en est fait mention dans la $9^{e}$ édition (en cours de parution depuis 1992) pour dire qu'elle n'est pas acceptable: «Loc. conj. Après que, suivi de l'indicatif ou du conditionnel, mais non du subjonctif. À la suite du moment où.» (DAF, 1992). Ici l'indication est minimale et l'usager doit se contenter de cet interdit sans explication.

\subsubsection{Multidictionnaire de la langue française}

Le Multidictionnaire de la langue française, dictionnaire de difficultés produit au Québec, adopte aussi la position prescriptive attendue, en distinguant la locution avant que, qui " exige le subjonctif » de la locution après que, qui « est suivie de l'indicatif ». 
Cette locution conjonctive [après que], qui exprime une notion réalisée, est suivie de l'indicatif, alors que la locution avant que, qui exprime une notion non encore réalisée, exige le subjonctif. Après que la marchandise aura été livrée, mais avant que la marchandise soit livrée. (p. 129)

Une possible confusion du mode verbal à utiliser à la suite d'après que est ici plus sousentendue qu'exprimée. À peine plus loquace que la $9^{e}$ édition du DAF, le Multidictionnaire n'exemplifie pas la forme fautive.

\subsubsection{Le Petit Robert de la langue française}

La construction après que avec le mode subjonctif est présente dès la première édition du Petit Robert (1967), et est dite moderne et critiquée. La marque " moderne » suppose ici que l'on a affaire à une nouvelle construction, qui entre en concurrence avec la construction plus traditionnelle après que + indicatif. L'emploi est cependant dit «critiqué» puisqu'il ne serait pas encore entré dans la norme. Cette indication normative n'empêche pas le dictionnaire d'illustrer la construction à l'aide de deux citations d'auteurs reconnus : André Gide et Henry de Montherlant.

Mod. (emploi critiqué) Après que et le subjonctif. « après que Vincent eût fermé sa porte" (Gide). "Trois semaines après que cette phrase ait été écrite " (Montherlant). (PR1967)

À partir de la refonte de 1993, le Petit Robert propose une justification pour cet emploi du subjonctif en indiquant qu'il se ferait par analogie avec avant que. Près de 30 ans plus tard, alors que cet emploi s'est confirmé dans l'usage et qu'il est maintenant accepté par plusieurs grammaires et dictionnaires (dont certains publiés aux éditions Le Robert) le traitement est toujours le même dans le Petit Robert : la construction après que + subjonctif reste critiquée dans le millésime 2021 de ce dictionnaire en un volume :

MOD. (emploi critiqué) Après que (suivi du subj. par analogie avec avant que) « après que Vincent eût fermé sa porte » (Gide). « Trois semaines après que cette phrase ait été écrite » (Montherlant). (PR2021)

\subsubsection{Grand Larousse de la langue française}

Tout comme Le Petit Robert, le Grand Larousse illustre l'emploi de après que + subjonctif au moyen de la citation d'un grand auteur (ici François Mauriac), mais signale qu'il n'est pas standard, en indiquant que la « construction n'est pas admise dans la langue soignée ». Aucune indication grammaticale n'est donnée, ni pour expliquer ni pour condamner la construction nouvelle.

Après que loc. conj. (suivie de l'indicatif) À la suite du moment où : Après qu'ils eurent dîné, ils partirent. REM. Le subjonctif tend à se répandre dans les propositions introduites par après que: Un siècle et demi après que cette parole ait été prononcée, nous savons que le bonheur en Europe est une illusion perdue (Mauriac). Cette construction n'est pas admise dans la langue soignée. (GLLF, 1971-197815)

Pourtant, ce dictionnaire en sept volumes est reconnu comme le premier vrai dictionnaire de langue de la maison Larousse. «Dès la préface, l'aspect linguistique et grammatical du dictionnaire est revendiqué par les auteurs, des linguistes de haute compétence » (Bonicel, 2005 : 45). Peut-être était-il trop tôt, dans les décennies 1970 et 1980, pour oser entériner une construction jusque-là jugée fautive. On sent pourtant une hésitation dans le traitement entre une approche descriptive, basée sur l'usage et l'ajout d'une citation littéraire, et une approche prescriptive, qui indique la nonadmission de la construction dans la langue soignée. Rappelons que le Grand Larousse, 
et ce dès sa première édition (1971-1978), a attesté la construction après que + subjonctif, construction qui est toujours absente du Petit Larousse illustré 50 ans plus tard.

\subsubsection{Dictionnaire des difficultés de la langue française, éditions Larousse}

Toujours chez la maison Larousse, le Dictionnaire des difficultés de la langue française d'Adolphe V. Thomas est un peu plus explicite. Après avoir condamné l'emploi du subjonctif à la suite de la locution après que, il indique qu'à la différence d'avant que, qui « annonce un fait futur », après que doit être suivi de l'indicatif puisqu'il « annonce un fait accompli, passé ».

Après que se construit avec l'indicatif ou le conditionnel (et non le subjonctif) : Après qu'ils eurent dîné (et non qu'ils eussent). Après que sœur Lætitia m'eut fait ma piqûre (Jacques Borel, L'Adoration, 143). Après que vous auriez parlé, que vous avez eu parlé, que vous eûtes parlé (Acad.). (Avant que veut le subjonctif, ce qui s'explique puisqu'il annonce un fait futur, donc éventuel, alors qu'après que annonce un fait accompli, passé.) (Thomas, $2012: 35$ )

\subsection{Le subjonctif n'est pas critiqué}

Dans les dictionnaires de notre corpus, c'est le Trésor de la langue française qui le premier atteste sans la critiquer la construction après que + subjonctif.

\subsubsection{Trésor de la langue française informatisé (TLFi)}

Le TLFi traite de la même façon les constructions après que suivi de l'indicatif et après que suivi du subjonctif, c'est-à-dire sans définition, sans renvoi, et en indiquant à la suite de chacune des constructions une série de quatre citations. Pour après que + subjonctif, les auteurs cités sont François Mauriac (1923), Colette (1928), Pierre Drieu la Rochelle (1939) et Albert Camus (1953).

En plus de ces auteurs reconnus, le TLFi appuie son acceptation de la construction avec subjonctif par une longue remarque à teneur grammaticale qui cite les explications de deux linguistes réputés : le Français Jean Stéfanini et le Belge Marc Wilmet.

Rem. Pourquoi le développement du subj. avec après que dep. le début du XX ${ }^{\mathrm{e}} \mathrm{s}$.? M. Stéfanini, en 1953, ds Remarques sur la syntaxe d'après que en fr. mod. estimait que chez les sujets qui emploient tantôt l'ind., tantôt le subj. avec après que, le subj. "exprime un procès dont la réalité ne peut faire aucun doute, dont la réalisation est garantie par après que, mais il permet de le considérer dans le temps in fieri, qui est en train de se faire pour aboutir au temps in esse, où se déroule (s'est déroulé ou se déroulera) le procès principal." (p. 79)." En se faisant suivre du subjonctif, après que conserve, dans toute la mesure du possible, sa symétrie avec avant que qui, grâce au discordantiel pouvait déjà établir entre deux faits un lieu non seulement temporel, mais idéel." (p. 83). En 1955, ds Nouvelles remarques sur la syntaxe d'après que, il s'efforce d'expliquer pourquoi un vaste public a manifesté un tel empressement à adopter la nouvelle tournure. [...]

Pour M. Wilmet, ds Après que suivi du subj., 1969, après que, "oblige l'usager du fr. mod. à saisir l'événement subordonné par sa séquelle verbale. Comme les tiroirs composés de l'indicatif ne suffisent plus à marquer l'aspect extensif, l'habitude s'installe de recourir au mode subjonctif, 一 où les formes simples et les formes composées constituent toujours des couples aspectuels véritables - , en sacrifiant ainsi l'expression du temps (au demeurant assurée par la conjonction) à celle de l'aspect (...) Le mode subjonctif, presque insensible à la notion de temps, fournit (...) 
une solution unique joignant la simplicité à l'efficacité.» pp. 37-38. (TLFi, 1971-1994)

Ce dernier exemple plus que tout autre démontre a contrario que lorsque l'espace est réduit et qu'il faut synthétiser la description d'un emploi en cours de réanalyse, les dictionnaires en un volume optent soit pour taire le débat (PLI2021), soit pour indiquer l'instabilité de la nouvelle construction en la disant critiquée, mais en l'appuyant de citations littéraires (PR2021).

Quand l'espace est suffisant et que les lexicographes supposent que leur lectorat est assez érudit et intéressé pour comprendre les nuances d'une langue en constante évolution, un emploi ailleurs tu ou critiqué est accepté, expliqué, justifié et très souvent illustré à l'aide de citations littéraires.

\subsubsection{Le Grand Robert de la langue française}

À la suite du TLFi, le Grand Robert entérine la construction après que + subjonctif en s'appuyant à la fois sur une remarque grammaticale qui fait la part belle à l'usage, et sur les citations d'auteurs français célèbres, André Gide et François Mauriac. Cette description était absente de la première édition de l'ouvrage (et de ses réimpressions).

Mod. APRÈs QUE, suivi du subj.

REM. Cet emploi, déjà signalé par Richelet ${ }^{16}$ et critiqué, tend à se répandre, concurremment à la construction à l'indicatif. Il apparaît ainsi comme le symétrique des tours introduits par avant que...: la distinction aspectuelle entre le procès en devenir (avant qu'il ne vienne) et la valeur accomplie (après qu'il soit parti), l'emporte ainsi sur l'opposition logique entre le fait supposé ou l'acte projeté (avant qu'il soit trop tard..., avant que je ne vienne) et le procès réel, puisque accompli (après qu'il a fini...).

Elle était restée, après que Vincent eût refermé sa porte sur elle, effondrée sur les marches. GIDE, les Faux-monnayeurs, p. 53.

Un siècle et demi après que cette parole ait été prononcée, nous savons que le bonheur en Europe est une illusion perdue. F. MAURIAC, le Cahier noir, p. 27-28. (GR2001 et GR2017)

\subsubsection{Dictionnaire des difficultés du français, éditions Le Robert}

Alors que plusieurs des dictionnaires expliquent l'emploi «fautif» du subjonctif avec après que par une confusion avec la construction acceptée pour avant que, Jean-Paul Colin fait l'inverse dans son Dictionnaire des difficultés du français; il se sert du modèle d'avant que pour justifier l'emploi du subjonctif avec après que. Ce dictionnaire renouvelle aussi les citations attestant après que + subjonctif, et, les multipliant, il rend la construction difficile à contester. Utilisée par tant de grands auteurs, elle est indubitablement entrée dans la langue, et dans un registre standard, sinon soigné.

Avant que étant régulièrement suivi du subjonctif, on finit par construire, par analogie, après que et avant que avec le même mode. Après qu'ils se fussent présentés l'un à l'autre (Aragon). Deux ans après que j'aie raconté les avatars et la rupture d'une amitié (Beauvoir). Après que Ruaux les ait dûment prévenus de l'expérience (Bazin). Une seconde après que le Christ soit revenu dans toute sa gloire (Cesbron). Après que nous ayons bu (Pons). Après qu'il ait été blessé (Mauriac). Après que nous ayons bien ri (Vialar). (Colin, 2012 : 35) 


\subsubsection{Dictionnaire des difficultés du français, Éditions De Boeck supérieur}

Sur un tout autre ton, les auteurs du Dictionnaire des difficultés du français, Joseph Hanse, et à sa suite Daniel Blampain, semblent céder à contre-cœur face à l'usage. Ils concèdent qu'il n'est plus possible de condamner l'emploi de la construction après que + subjonctif maintenant que les grammairiens la «tolèrent » et qu'elle est fréquemment employée "même chez les écrivains». Ce faisant, ils valident cette nouvelle construction en s'appuyant sur trois autorités en matière de langue : la grammaire, l'usage et la littérature.

Après que réclame l'indicatif dans tous les cas. C'est à notre époque seulement que le subjonctif est devenu en assez peu de temps une habitude dans la presse, à la radio, dans l'usage courant et même chez les écrivains. Au point que des grammairiens, ne tenant pas compte des exemples infiniment plus nombreux de l'emploi de l'indicatif dans les milieux cultivés et dans la langue littéraire, tolèrent le subjonctif au nom de l'usage et tentent même de le justifier au nom de la logique et de l'histoire de la langue.

$[$...]

L'indicatif est certainement à recommander, mais l'on ne taxera pas d'ignorance ceux qui, influencés par un large usage écrit et oral, emploient le subjonctif. (Hanse et Blampain, 2012 : 54)

\subsection{Des dictionnaires inconstants}

Ce rapide survol de la description faite de la construction après que + subjonctif par quelques dictionnaires contemporains permet de constater une grande diversité dans les positions proposées. Nous pouvons noter que plus l'ouvrage consulté fait de place à une analyse grammaticale, plus il sera enclin à justifier l'emploi de la nouvelle construction. De même, à l'intérieur d'une même maison d'édition, les dictionnaires de difficultés ou les dictionnaires en plusieurs volumes seront plus explicites et plus accueillants pour ce type d'évolution de la langue que les dictionnaires en un volume qui ne disposent pas de l'espace nécessaire pour entrer dans les nuances et qui doivent être rapidement décodables par un public néophyte. Pour le cas étudié ici, le Trésor de la langue française, produit au $\mathrm{XX}^{\mathrm{e}}$ siècle, est donc beaucoup plus à jour et éclairant que des dictionnaires en un volume millésimés de 2021.

\section{Description d'après que dans le dictionnaire Usito}

Nous avons constaté que le traitement réservé à la conjonction complexe après que différait selon les dictionnaires, qu'ils soient de langue ou de difficultés, monovolumaires ou plurivolumaires, et ce parfois à l'intérieur d'une même maison d'édition. Les paramètres expliquant les différences seraient principalement la place accordée aux explications grammaticales dans les ouvrages étudiés, et l'espace disponible pour fournir les explications nécessaires, faisant des dictionnaires en un volume les plus mal lotis de notre corpus bien que les plus récents.

La lexicographie, comme la langue, est en constante évolution. Les dictionnaires conçus en format numérique peuvent se permettre une utilisation de l'espace plus souple que les dictionnaires d'abord conçus en format papier. C'est le cas du dictionnaire québécois Usito ${ }^{17}$, dont la nomenclature est comparable à celle du Petit Robert (environ 60000 entrées), mais qui n'est disponible qu'en ligne et conçu sur support numérique. 
Ce dictionnaire est significativement riche en indications grammaticales présentées dans des infobulles ou en remarques (voir Vincent, 2021). En respect de sa ligne éditoriale, Usito vise à indiquer la norme plutôt qu'à la prescrire, et se soucie d'informer l'usager autant pour l'encodage que pour le décodage et la révision. En tant que membres de l'équipe du dictionnaire, nous avons proposé d'y attester la construction après que + subjonctif, en l'accompagnant de citations tant françaises que québécoises, et en indiquant qu'elle est encore critiquée dans certains ouvrages de référence (grammaires ou dictionnaires). Usito présente maintenant la description suivante :

Conj. de subordination complexe APRÈs QUE (+ indic. ou + subj.). Après que la décision a été prise ou après que la décision ait été prise. Après que les enfants se sont couchés ou après que les enfants se soient couchés. "Nous avons enfin touché terre quelques heures après que le brouillard s'est levé " (N. Audet, 2002). "Après que le prévenu ait été déclaré coupable, le juge décide, en fonction de ce rapport, du type de probation à appliquer.» (Le Figaro, 2013)

REM. La construction après que (+ subj.), encore critiquée par certaines sources, est aujourd'hui d'usage courant dans toute la francophonie.

\section{Conclusion}

Le parcours historique des grammaires et des dictionnaires a permis de constater que le choix du mode à la suite de après que n'a pas été soumis à des tensions d'évolution avant le $\mathrm{XX}^{\mathrm{e}}$ siècle. Les mentions insistant sur le mode requis par après que apparaissent à ce moment, mais elles n'abordent pas encore l'emploi du subjonctif. Excepté l'avis étonnant de Richelet (1680) et des ouvrages qui - selon toute vraisemblance - s'en sont inspirés, c'est à partir des années 1930 que l'usage du subjonctif est présenté et critiqué dans les grammaires. Après des jugements sévères jusque dans les années 1960, les grammairiens et lexicographes lâchent du lest. Grevisse amorce le mouvement dès les années 1950, le Petit Robert dans les années 1960. Ce n'est ensuite que dans les années 1970 (Dubois et Lagane 1973, TLF 1971-1994) et 1980 (Grevisse 1980) que le basculement est atteint.

Tant dans les grammaires que dans les dictionnaires, le volume des ouvrages et le public auquel ils s'adressent influent sur la position adoptée. Plus l'ouvrage est bref et destiné à un public scolaire, plus la sanction normative est forte. La vision de la norme y est manichéenne. Il faut dire que le continuum des usages en synchronie et leur évolution en diachronie peuvent être déstabilisants pour un public moins averti, non linguiste. D'autres ouvrages naviguent entre condamnation du bout des lèvres et constat d'un usage en pleine progression, preuve que l'évolution n'est pas totalement aboutie et que les usages coexistent. Il reste, bien sûr, des irréductibles de l'indicatif, mais peuvent-ils encore se permettre de ne laisser aucune place au subjonctif ? Nous espérons avoir montré que non.

Avant de clore cette recherche dans les ouvrages de référence, il resterait à dire quelques mots sur les raisons de l'évolution vers le subjonctif. Il nous est toutefois impossible, sans déborder des objectifs de l'étude proposée ici et de l'espace imparti, de traiter véritablement de la question. Nous ne pouvons que l'évoquer, à la suite notamment de Guillaume (1929), Wilmet (1969), Dolbec et Le Flem (1981), Canut et Ledegen (1998). La raison qui est le plus souvent invoquée est une analogie avec avant que (analogie qui n'a d'ailleurs pas influé sur quand ou lorsque). Toutefois, il est étonnant 
que le subjonctif, morphologiquement plus complexe, ait été un attracteur. La confusion homographique de certaines formes du passé antérieur et du subjonctif plusque-parfait, tous deux temps de l'écrit en perte de vitesse, est aussi remise en doute (Grevisse-Goosse 2016): comment l'écrit aurait-il ainsi influencé l'oral? Chevalier et al. (1962) et Wilmet (2010) proposent l'explication la plus plausible : au moment où le passé composé prend le relais du passé simple dans la principale, la concordance aspectuelle imposerait un temps surcomposé dans la subordonnée. Cette forme souvent jugée suspecte aurait alors été remplacée par le subjonctif passé dont la valeur aspectuelle permet d'insister sur l'accompli, en dehors de toute référence à une époque, simplifiant dès lors le système de la concordance des temps.

\section{BIBLIOGRAPHIE}

ACADÉMIE FRANÇAISE, 1932-1935 ; 1992-, Dictionnaire de l'Académie française, $8^{\mathrm{e}}$ et $9^{\mathrm{e}}$ éditions, Paris.

ARRIVÉ, M., GADET, F., GALMICHE, M., 1986, La grammaire d'aujourd'hui. Guide alphabétique de linguistique française, Paris, Flammarion.

BAYOL, M.-C., BAVENCOFFE, M.-J., 2013 [1995], La grammaire française, Paris, Nathan.

BERTRAND-SAVARD, S., BEAULIEU, S., CAYOUETTE, M.-M., MINET, C., 2013, La nouvelle grammaire en contexte, Anjou, CEC.

BESCHERELLE, 2006, La grammaire pour tous, Montréal, Hurtubise HMH.

BESCHERELLE, L.-N., 1853, Dictionnaire national, Paris, Simon.

BLAMPAIN, D., HANSE, J., 2012, Dictionnaire des difficultés du français, Bruxelles, De Boeck supérieur, $6^{\mathrm{e}}$ édition.

BOIVIN, M.-C., PINSONNEAULT, R., 2019 [2008], La grammaire moderne. Description et éléments pour sa didactique, Montréal, Beauchemin-Chenelière éducation.

BONENFANT Ch., avec la coll. de TURCOTTE G., 2008, Boite à outils, Mont-Royal, Modulo.

BONICEL L., 2005, « Le Grand Larousse de la langue française (1971-1978) : de l'innovation lexicographique à l'échec dictionnairique ", Éla. Études de linguistique appliquée, 137, p. 39-49.

BOSQUART, M., 1998, Nouvelle grammaire française, Montréal, Guérin.

CANUT, C. et LEDEGEN, G., 1998, « Après que ou la fluctuation des modes en français parlé ", Langage et société, 85, p. 25-51.

CHARTRAND, S.-G. et al., 1999, Grammaire pédagogique du français d'aujourd'hui, Montréal, Graficor.

CHERDON, Ch., 2005 [1985], Guide de grammaire française, Bruxelles, De Boeck.

CHEVALIER, J.-C. et al., 2002 [1964], Grammaire du français contemporain, Paris, Larousse.

CHRISTENSEN, M.-H., FUCHS, M., KORACH, D., 1995, Grammaire alphabétique. Rééditée en 2005. Paris, Le Robert \& Nathan. 
CHRISTOPHE, P., 2013, La grammaire française en fiches, Paris, Ellipses.

COLIN, J.-P., 2006, Dictionnaire des difficultés du français, Paris, Éditions Le Robert.

Corpus de textes linguistiques fondamentaux. [En ligne] http://ctlf.ens-lyon.fr.

DELATOUR, Y., JENNEPIN, D., LÉON-DUFOUR, M., 2004, Nouvelle grammaire du français, Paris, Hachette.

DENIS, D., SANCIER-CHATEAU, A., 1997 [1994], Grammaire du français, Paris, Le livre de Poche.

DOLBEC, J. et LE FLEM, C., 1981, « Le subjonctif avec « après que » : faute ou variation significative ? », Cahier de linguistique, 7, p. 123-154.

DUBOIS, J., LAGANE, R., 2004 [1973], Nouvelle grammaire du français, Paris, Larousse.

ÉLUERD, R., 2004 [2002], Grammaire descriptive de la langue française, Paris, Armand Colin.

ÉLUERD, R., 2009, La grammaire française, Paris, Garnier, Littré.

FAIRON, C., SIMON, A.-C., 2018, Le petit Bon usage, Louvain-la-Neuve, De Boeck Supérieur.

FÉRAUD, J.-F., 1787-1788, Dictionnaire critique de la langue française, Marseille, Mossy.

FOURNIER, N., 1988, Grammaire du français classique, Paris, Belin.

GARAGNON, A.-M., CALAS, F., 2004 [2002], La phrase complexe, Paris, Hachette supérieur.

GOBBE, R., TORDOIR, M., 2005 [1984], Grammaire française, Bruxelles, Plantyn.

GREVISSE, M., GOOSSE, A., 1995 [1980], Nouvelle grammaire française, Bruxelles, De Boeck.

GREVISSE, M., GOOSSE, A., 2016 [1986], Le bon usage, Louvain-La-Neuve, De Boeck Supérieur.

GUILLAUME, G., 1929, Temps et verbe, Paris, Champion.

LAPORTE, M., ROCHON, G., 2011 [2007], Nouvelle grammaire pratique pour tous, Anjou, CEC.

LAURENT, N., DELAUNEY, B., 2012, Bescherelle. La grammaire pour tous, Paris, Didier Hatier.

LE GOFFIC, P., 1993, Grammaire de la phrase française, Paris, Hachette supérieur.

Le Grand Larousse de la langue française, 1971-1978, Paris, Éditions Larousse.

Le Grand Robert de la langue française, 1969, 2001, 2017, Paris, Éditions Le Robert.

Le Nouveau Petit Robert : dictionnaire alphabétique et analogique de la langue française, 1993, Paris, Éditions Le Robert.

Le Petit Larousse illustré 2021, 2020, Paris, Éditions Larousse.

Le Petit Robert de la langue française 2021, 2020, Paris, Éditions Le Robert.

LECAVALIER, J., avec la coll. de J. BONNEVILLE, 2013 [2006], L'express grammatical, Saint-Laurent, ERPI.

LEFRANÇOIS, P., 2020 [2013], Français écrit pour futurs enseignants, Montréal, JFD.

LITTRÉ, É., 1873-1874, Dictionnaire de la langue française, Paris, Hachette.

MAINGENEAU, D., 2007 [1991], Précis de grammaire pour les concours, Paris, Armand Colin.

NARJOUX, C., 2018, Le Grevisse de l'étudiant, Louvain-la-Neuve, De Boeck Supérieur.

NICOT, J., 1606, Thresor de la langue francoyse, tant ancienne que moderne, Paris, Douceur.

PELLAT, J.-C., FONVIELLE, S., 2016, Le Grevisse de l'enseignant, Paris, Magnard. 
PIRON, S., 2017, Grammaire française. Vol. 1 et 2, Louvain-la-Neuve, De Boeck Supérieur.

PORÉE, M.-D., 2011, La grammaire française pour les nuls, Paris, First-Gründ.

RIEGEL, M., PELLAT, J.- Ch., RIOUL, R., 2018 [1994], Grammaire méthodique du français, Paris, PUF.

STÉFANINI, J. 1953, « Remarques sur la syntaxe d"après que' en français moderne », Annales de la Faculté des lettres d'Aix, 27, p. 65-87.

STRUVE-DEBEAUX, A., 2010, Maîtriser la grammaire française, Paris, Belin.

THOMAS, A.V., 2012, Dictionnaire des difficultés de la langue française, Paris, Éditions Larousse.

Trésor de la langue française informatisé, 1971-1994, Nancy. [en ligne : http://atilf.atilf.fr/tlf.htm]

VASSEVIÈRE, J., 2013 [2009], Bien écrire pour réussir ses études, Paris, Armand Colin.

VERJANS, Th., 2013, La subordination, Paris, Armand Colin.

VILLERS, M.-É. de, 2015, Multidictionnaire de la langue française, $6^{\mathrm{e}}$ édition, Montréal, Québec/ Amérique.

VILLERS, M.-É. de, 2009 [2003], La nouvelle grammaire en tableaux., Montréal, Québec Amérique.

VINCENT, N., 2021, «Quand le lexicographe s'adresse à l'usager. Regard sur l'utilisation des remarques dans deux dictionnaires généraux du français du XXI ${ }^{\mathrm{e}}$ siècle », dans $\mathrm{C}$. Jacquet-Pfau «La vulgarisation dans les dictionnaires et encyclopédies », La Linguistique, n 57, p. 133-152.

WILMET, M., 1969, «'Après que’ suivi du subjonctif », La linguistique, 5-2 : 27-39.

WILMET, M., 2010 [1997], Grammaire critique du français, Bruxelles, De Boeck.

\section{NOTES}

1. Nous remercions chaleureusement Jean-Claude Klein, l'un des 400 étudiants présents, pour avoir partagé cette anecdote avec nous.

2. Les deux auteures de cet article sont des collaboratrices du dictionnaire Usito.

3. Nous avons parfois consulté plusieurs éditions d'un même ouvrage. Celui-ci ne compte alors que pour un seul titre dans le corpus. Pour des raisons d'espace, nous ne sommes pas en mesure de donner les références complètes de ces ouvrages. Elles peuvent être consultées sur le site du CTLF ou de la BnF.

4. Meigret (1550), Estienne (1557), Ramus (1572), Bosquet (1586), Maupas (1607), Oudin (1632), Vaugelas (1647), Irson (1656), Arnauld et Lancelot (1660), Buffier (1709), Valange (1721), Gaullyer (1722), Girard (1747), Panckoucke (1749), Wailly (1759), Bertera (1773), Royon (1777), De Prunay (1777), Domergue (1778), Chompré et al. (1778), Adam (1779), Lhomond (1780), Blondin (1787), Fabre (1787), Bauchaint (1789), Chemin-Dupontès (1794), Jacquemard (1795), Letellier (1805), Lemare (1807), Girault-Duvivier (1811), Noël et Chapsal (1823), Serreau et Boussi (1824), Boniface (1829), Vanier (1836), Larousse (1849), Guérard (1851), Leclair et Rouzé (1874), Larive et Fleury (1875), Brachet et Dussouchet (1883), Chassang (1888), Crouslé (1888), Clédat (1894), Crouzet, Berthet et Galliot (1909), Bauche (1828), Frei (1929), Aumeunier et Zeyaco (1937), Galichet (1947).

5. Chiflet (1659), Vairasse d'Allais (1681), Régnier-Desmarais (1705), Restaut (1730), Vallart (1744), Beauzée (1767), Boinvilliers (1818), Bescherelle (1834), Landais (1835), Brelet (1899), Sudre (1907), Bruno et Bony (1908), Maquet et al. (1908), Calvet et Chompret (1917), Lanusse et Yvon (1920), Radouant (1922), Académie (1932), Bally (1932), Michaut et Schricke (1934), Grevisse (1936), Gaiffe et al. (1936), Bruneau et Heulluy (1937), Bloch et Georgin (1937), Gougenheim (1938), Le Bidois et 
Le Bidois (1938), Dauzat (1947), Von Wartburg et Zumthor (1947), De Boer (1947), Cayrou (1948), Souché (1948), Wagner et Pinchon (1961), Chevalier et al. (1962), Dubois et Lagane (1973).

6. Nous avons également vérifié l'orthographe du passé simple, du passé antérieur, du subjonctif imparfait et plus-que-parfait dans les tableaux de conjugaison des grammaires du corpus : les temps sont bien différenciés par l'orthographe (eut, fut à l'indicatif; eust/eût, fust/fût au subjonctif), excepté chez Vallange (1721), qui propose une orthographe modernisée pour les deux modes : ut, fut.

7. Voir la partie consacrée aux dictionnaires et la note 14 .

8. À cet égard, les grammaires n'envisagent pas toujours les relations temporelles sous le même angle. Elles considèrent soit le sens intuitif du subordonnant pour qualifier la subordonnée (après que est alors dans une subordonnée de postériorité, cf. Sudre 1907), soit la position relative du procès de la subordonnée par rapport à celui de la principale (après que est alors dans une subordonnée d'antériorité, cf. Radouant 1922).

9. D'après Dolbec et Le Flem (1981), Guillaume (1929) a déjà fait part de l'emploi du subjonctif, dans son ouvrage Temps et verbe. Puisqu'il s'agit d'une étude plus spécifique et non d'un ouvrage de linguistique générale, il ne fait pas partie de notre corpus.

10. Les éditions suivantes de 1944 et de 1965 ont seulement changé le verbe de la première phrase, s'acoquiner est devenu se confondre: «Voici qu'à son tour l'imparfait du subjonctif se confond avec le passé simple de l'indicatif à la $3^{\text {e }}$ sing. » (Bally 1944 et 1965 [1932] : 259, par. 419).

11. Les 16 éditions ne comptent que pour deux titres au sein des 117 du corpus, le premier avec les éditions antérieures à 1986 et le second à partir de 1986.

12. Porée (2011), Bonenfant (2013), Verjans (2013), Christophe (2013), Bertrand-Savard (2013), Lefrançois (2020).

13. Garagnon et Calas (2004), Éluerd (2004), Christensen et al. (2005), Bescherelle (2006), de Villers (2009), Laporte et Rochon (2011), Bayol et Bavencoffe (2013), Lecavalier (2013), Boivin et Pinsonneault (2019).

14. Voir la note 14 à la section 3.3.2.

15. L'article est resté inchangé dans l'édition 1986 (réimprimée en 1989) du Grand Larousse de la langue française.

16. La première édition du dictionnaire de Richelet indique «Après que. Conjonctive qui régit l'indicatif, et le subjonctif. [Après que Salomon eut bâti à Dieu un Temple, il se bâtit un Palais pour lui. Port Roial.] » (1680). Nous n'avons pas trouvé la citation dans la grammaire de Port-Royal (1660), mais c'est surtout un exemple au passé antérieur. Cette description (avec l'exemple à l'indicatif) est reprise pratiquement mot à mot dans la $2^{\mathrm{e}}$ édition du dictionnaire de Furetière (alors qu'elle n'est pas dans la première, parue en 1690) : « APRÈs. Conjonctive qui régit l'indicatif et quelquefois le subjonctif. Après que Salomon eut bâti un Temple à Dieu, il se bâtit un Palais pour lui. Port-R. Après qu'on est sorti du péril, on ne songe plus au Saint. » (Furetière, 1702), puis dans les différentes éditions du Dictionnaire de Trévoux au cours du XVIII ${ }^{\mathrm{e}}$ siècle. Ces affirmations sur le subjonctif, qui n'est justement pas attesté dans les exemples proposés, très probablement toutes issues de Richelet, étonnent alors qu'aucune grammaire de la même époque n'atteste la possibilité de ce mode verbal à la suite d'après que. Une analyse de corpus des XVII ${ }^{\mathrm{e}}$ et XVIII ${ }^{\mathrm{e}}$ siècles, menée par Fournier (1998), n'a d'ailleurs permis de trouver aucun exemple de après que suivi du subjonctif : « Notre corpus classique ne nous a fourni que des exemples à l'indicatif. » Fournier (1998 : 357). Les autres dictionnaires anciens qui exemplifient le mode à la suite de après que (Nicot 1606, Furetière 1690, Féraud 1787-1788, Bescherelle 1856 et Littré 1873-1877) ne consignent que l'indicatif et le font dans des contextes non ambigus.

17. https://usito.usherbrooke.ca 


\section{RÉSUMÉS}

L'article porte sur la règle d'emploi du mode après le subordonnant après que telle qu'elle est consignée dans les grammaires et dictionnaires depuis le $\mathrm{XVI}^{\mathrm{e}}$ siècle. La règle traditionnelle appelle l'indicatif, mais le subjonctif s'est immiscé dans l'usage au $\mathrm{XX}^{\mathrm{e}}$ siècle. Initialement vivement critiqué, il est de plus en plus présenté et jugé acceptable par les ouvrages de référence.

The paper examines the rule for choosing mode after après que, as it has been recorded in grammars and dictionaries since the $16^{\text {th }}$ century. The traditional rule is to use the indicative, but the subjunctive is more and more widely used since the $20^{\text {th }}$ century. Initially strongly criticized, it is more and more presented and said acceptable by grammars and dictionaries.

\section{INDEX}

Keywords : après que, indicative, subjonctive, grammars, dictionnaries

Mots-clés : après que, indicatif, subjonctif, grammaires, dictionnaires

\section{AUTEURS}

SOPHIE PIRON

Université du Québec à Montréal

\section{NADINE VINCENT}

Université de Sherbrooke 